\title{
EGFR Primary T790M and L858R Double Mutation Confers Clinical Benefit to Erlotinib and Resistance to Osimertinib in One Lung Adenocarcinoma Patient: A Case Report
}

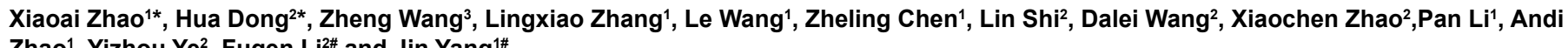
Zhao', Yizhou $\mathrm{Ye}^{2}$, Fugen $\mathrm{Li}^{2 *}$ and Jin Yang ${ }^{1 \#}$

${ }^{1}$ Department of Medical Oncology, The First Affiliated Hospital of Xian Jiaotong University, Xi'an, P.R. China

${ }^{2}$ The Research and Development Center of Precision Medicine, 3D Medicines Inc., Shanghai, P.R. China

${ }^{3}$ Informatics and Technology, Astra Zeneca, Shanghai, P.R. China

\#Contributed equally to the paper

\begin{abstract}
Introduction: EGFR TKI has been widely studied in both research and clinic. However, only few limited studies focus on EGFR primary resistance rather than acquired mutation. Usually, EGFR T790M mutation is resistant to first generation TKI (erlotinib, gefitinib) and sensitive to third generation TKI Osimertinib. Here, we report an EGFR primary T790M and L858R double mutation patient confers clinical benefit to Erlotinib and resistance to Osimertinib.
\end{abstract}

Case presentation: Here we present a 70 year-old woman with lung adenocarcinoma harboredprimary T790M and L858R EGFR double mutation and underwent multiple lines of treatments. She benefited from the firstgeneration of EGFR TKI Erlotinib, and later quickly developed resistance to the third-generation TKI Osimertinib after disease progression, then followed a few rounds of chemotherapies. Multiple resistant EGFR mutations were detected, indicating frequent complex tumor heterogeneity in later stage patients caused by subclone evolution. Colonies with distinct Osimertinib resistance mutations included well known EGFR mutations L792V, L718V/R, G796S/A, and novel EGFR G729V and D1014V mutations, which are predicted to be acquired osimertinib resistance mutations by 3D-structural remodeling.

Conclusion: Our study revealed that EGFR primary T790M accompany with L858R mutation could benefit from erlotinib, and has stable disease for 7 months. The patient also benefited from chemotherapy for three months after resistance to osimertinib. ctDNA-based assay is a valuable tool for late stage cancer diagnosis, monitoring and intervention.

Keywords: EGFR; Erlotinib; Osimertinib; Lung adenocarcinoma; Circulating tumor DNA assay

Abbreviations: EGFR: Epidermal Growth Factor Receptor; TKI: Tyrosine Kinase Inhibitors; NSCLC: Non-Small Cell Lung Cancer; ARMS-PCR: Amplification Refractory Mutation System Polymerase Chain Reaction; ddPCR: Droplet Digital Polymerase Chain Reaction; CT: Computerized Tomography; PET-CT: Positron Emission Tomography-Computed Tomography; ctDNA: Circulating tumor DNA; PFS: Progression-Free Survival; CEA: Carcinoembryonic Antigen

\section{Introduction}

The epidermal growth factor receptor (EGFR)-tyrosine kinase inhibitors (TKIs) include the previously widely used first-generation gefitinib and erlotinib, the second-generation afatinib and nowadays the third-generation osimertinib with remarkable activity in nonsmall cell lung cancer (NSCLC) patients [1,2]. The sensitive biomarkers, primary and acquired resistant mechasnism were summarized in Table 1 from literature [3-8]. Minor clones with the T790M mutation in treatment-naive tumors that contain classic sensitizing mutations were under estimated by conventional PCR and Sanger sequencing methods. Studies using more sensitive techniques have reported T790M mutations in $35 \%$ and $79 \%$ of EGFR-mutant, NSCLC pretreatment specimens and the T790M mutation in some cases were associated with longer progression-free survival to erlotinib $[9,10]$. Here we describe one patient who had primary L858R and T790M double mutation was sensitive to erlotinib and resistant to osimertinib, finally benefited from chemotherapy for three months after resistance to osimertinib.

\section{Case Presentation}

A 63-year-old famale, with ECOG performance status of 1 was diagnosed in June 2010 to be lung adenocarcinoma stage IB(pT2N0M0). The patient had no smoking history, and no family history of malignant tumor. ARMs PCR detected both L858R and T790M mutations in the tumor sample. Droplet digital PCR(ddPCR) for the same FFPE slides confirmed the initial ARMs PCR results with allele frequency 0.254 for L858R mutation and 0.0021 for T790M mutation (Supplementary Table 1). Adjuvant chemotherapy with 4 cycles of gemcitabine and Cisplatin were administrated after surgical resection (left pneumonectomy). No recurrence was seen until September, 2014.

*Corresponding author: Jin Yang, Department of Medical Oncology, The First Affiliated Hospital of Xian Jiaotong University, Xi'an, Shanxi 710061, P.R. China, Tel: +86-2985323112; E-mail: 1473106133@qq.com

Hua Dong, The Research and Development Center of Precision Medicine, 3D Medicines Inc., Shanghai, 201114, P.R. China, Tel: +86-29-85323338; E-mail: fugen.li@3dmedcare.com

Fugen $\mathrm{Li}$, The Research and Development Center of Precision Medicine, 3D Medicines Inc., Shanghai, 201114, P.R. China, Tel: +86-29-85323338; E-mail: wx89211@163.com

Received October 11, 2018; Accepted November 09, 2018; Published November 12, 2018

Citation: Zhao X, Dong H, Wang Z, Zhang L, Wang L, et al. (2018) EGFR Primary T790M and L858R Double Mutation Confers Clinical Benefit to Erlotinib and Resistance to Osimertinib in One Lung Adenocarcinoma Patient: A Case Report. J Cancer Sci Ther 10: 366-370. doi: 10.4172/1948-5956.1000569

Copyright: (c) 2018 Zhao X, et al. This is an open-access article distributed unde the terms of the Creative Commons Attribution License, which permits unrestricted use, distribution, and reproduction in any medium, provided the original author and source are credited. 
Citation: Zhao X, Dong H, Wang Z, Zhang L, Wang L, et al. (2018) EGFR Primary T790M and L858R Double Mutation Confers Clinical Benefit to Erlotinib and Resistance to Osimertinib in One Lung Adenocarcinoma Patient: A Case Report. J Cancer Sci Ther 10: 366-370. doi: $10.4172 / 1948-5956.1000569$

\begin{tabular}{|c|c|c|c|c|}
\hline Generation & Drug & Sensitive biomarkers & Primary resistance mechanism & Acquired resistance mechanism \\
\hline \multirow{2}{*}{$1^{\text {st }}$} & Gefitinib & EGFR L858R & \multirow{5}{*}{$\begin{array}{l}1 . \\
2 . \\
3 . \\
4 .\end{array}$} & \multirow{5}{*}{$\begin{array}{ll}\text { 1. Secondary EGFR mutations, major in EGFR T790M. } \\
\text { 2. } \\
\text { Gene copy alterations of alternative pathways including } \\
\text { MET or ERBB2 or MAPK amplification. } \\
\text { 3. Mutations in downstream effector molecules of EGFR } \\
\text { including KRAS, NRAS, BRAF V600E, PIK3CA } \\
\text { mutation. } \\
\text { 4. Other mechanism including EMT, SCLC transformation, } \\
\text { AXL or NF-kb activation et al. }\end{array}$} \\
\hline & Erlotinib & EGFR ex19del & & \\
\hline \multirow[b]{3}{*}{$2^{\text {nd }}$} & \multirow[b]{3}{*}{ Afatinib } & EGFR L858R & & \\
\hline & & EGFR ex19del & & \\
\hline & & $\begin{array}{l}\text { EGFR wildtype (Relative potent } \\
\text { compared to } 1^{\text {st }} \text { generation TKI) }\end{array}$ & & \\
\hline \multirow[b]{4}{*}{$3^{\text {rd }}$} & \multirow[b]{4}{*}{ Osimertinib } & EGFR L858R & \multirow{4}{*}{$\begin{array}{l}\text { 1. } \quad \text { EGFR Exon } 20 \text { insertions or duplications. } \\
\text { 2. } \text { EGFR germline BIM deletion. } \\
\text { 3. }\end{array}$} & \multirow{4}{*}{$\begin{array}{l}\text { 1. Secondary EGFR mutations, major in EGFR C797S/R, } \\
\text { L798I, G796S/R, L792F/H. } \\
\text { 2. Gene copy alterations of alternative pathways including } \\
\text { MET or ERBB2 or MAPK amplification. } \\
\text { 3. Mutations in downstream effector molecules of EGFR } \\
\text { including KRAS, NRAS, BRAF V600E, PIK3CA } \\
\text { mutation. } \\
\text { Other mechanism including EMT, SCLC transformation, } \\
\text { Src-AKT, Src-YAP1 activation et al. }\end{array}$} \\
\hline & & EGFR ex19del & & \\
\hline & & EGFR L858R+T790M & & \\
\hline & & EGFR ex19del+T790M & & \\
\hline
\end{tabular}

Table 1: Sensitive biomarkers, primary and acquired resistance mechanism for EGFR three generation TKIs.

Initial diagnosis

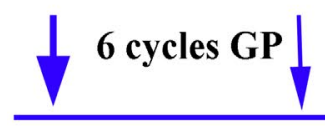

2010.6

(FFEP)

L858R+

T790M+

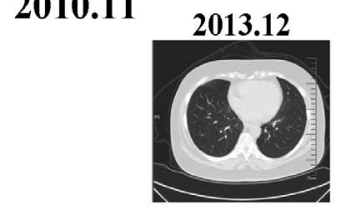

SD

Lung metastasis Start Erlotinib

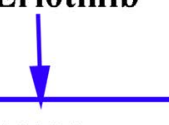

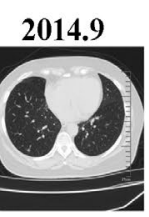

SD

Brain metastasis

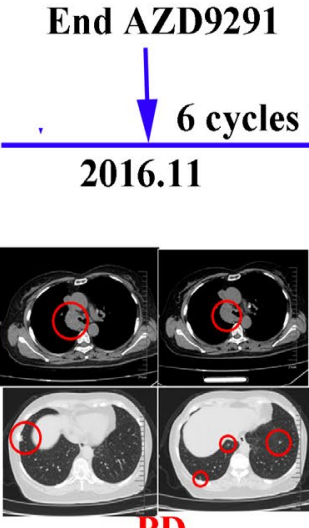

PD
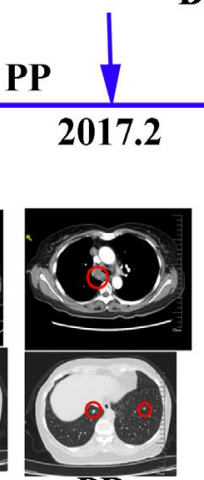

PR
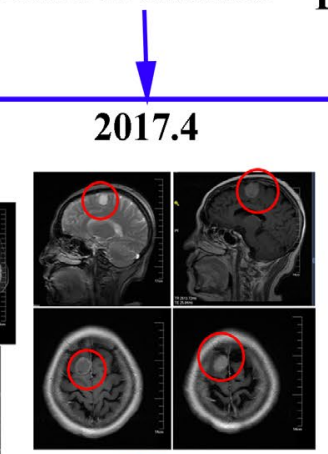

PD
2016.3

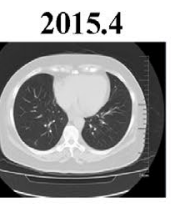

SD

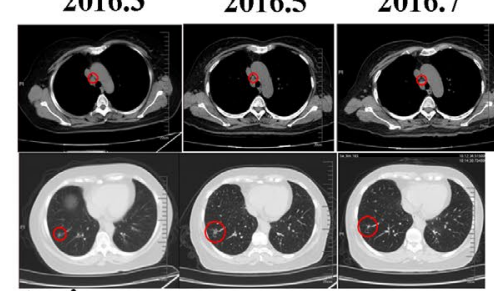

SD

(ctDNA) assay

L792V,G796A/S, L718V/R.....
2017.6

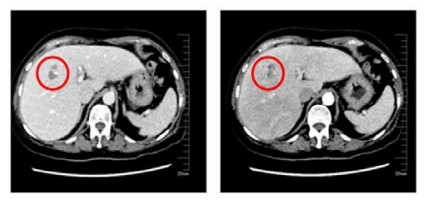

PD

Figure 1: Imaging, pathological examination of the case. Computed tomography of the chest showed tumor change during the treatment. Images of 2013.12 as baseline and the following 10 time points for the later treatments were shown; red boxes highlight the newly emerging nodule and serves as criteria for evaluation. RECIST standard was used for assessment of response.

In September 2014, computed tomography (CT) scan revealed multiple lung nodules. No metastasis was seen by abdominal ultrasound examination and magnetic resonance imaging (MRI). In Feburary 2016, positron emission tomography (PET)-computed tomography (CT) discovered the left clavicular upper and mediastinal multiple lymph node metastasis. She was treated with Erlotinib treatment since then, and stable disease was confirmed according to RECIST assessment. The patient had 7-month progression-free survival (PFS) (Figure 1). In September 2016, a nodular pattern in the left lung was emerged, and AZD9291 (trial agent at that time) was taken. In November 2016, the disease was progressed.
From November 2016, the patient had then been treated for six cycles of combination of Pemetrexed $0.8 \mathrm{~g}$ dwith Cis-Paclitaxel 40 $\mathrm{mg} \mathrm{d} 1-3, \mathrm{q} 3 \mathrm{w}$. The patient had partial response after two cycles of Pemetrexed/Cisplatin (PP) treatment and stable disease after four cycles. The disease control time is about 3 months. In April 2017, the cancer metastasized into brain and later liver. A plasma-based circulating tumor DNA (ctDNA) assay was performed in June 2017. The multiple heterogeneous EGFR mutations, such as L858R and T790M were detected and these mutations were also observed by ARMs PCR in the initial test. In addition, more EGFR mutations, such as L792V, G796A/S and L718V/R were also detected and those are well 
Citation: Zhao X, Dong H, Wang Z, Zhang L, Wang L, et al. (2018) EGFR Primary T790M and L858R Double Mutation Confers Clinical Benefit to Erlotinib and Resistance to Osimertinib in One Lung Adenocarcinoma Patient: A Case Report. J Cancer Sci Ther 10: 366-370. doi: $10.4172 / 1948-5956.1000569$

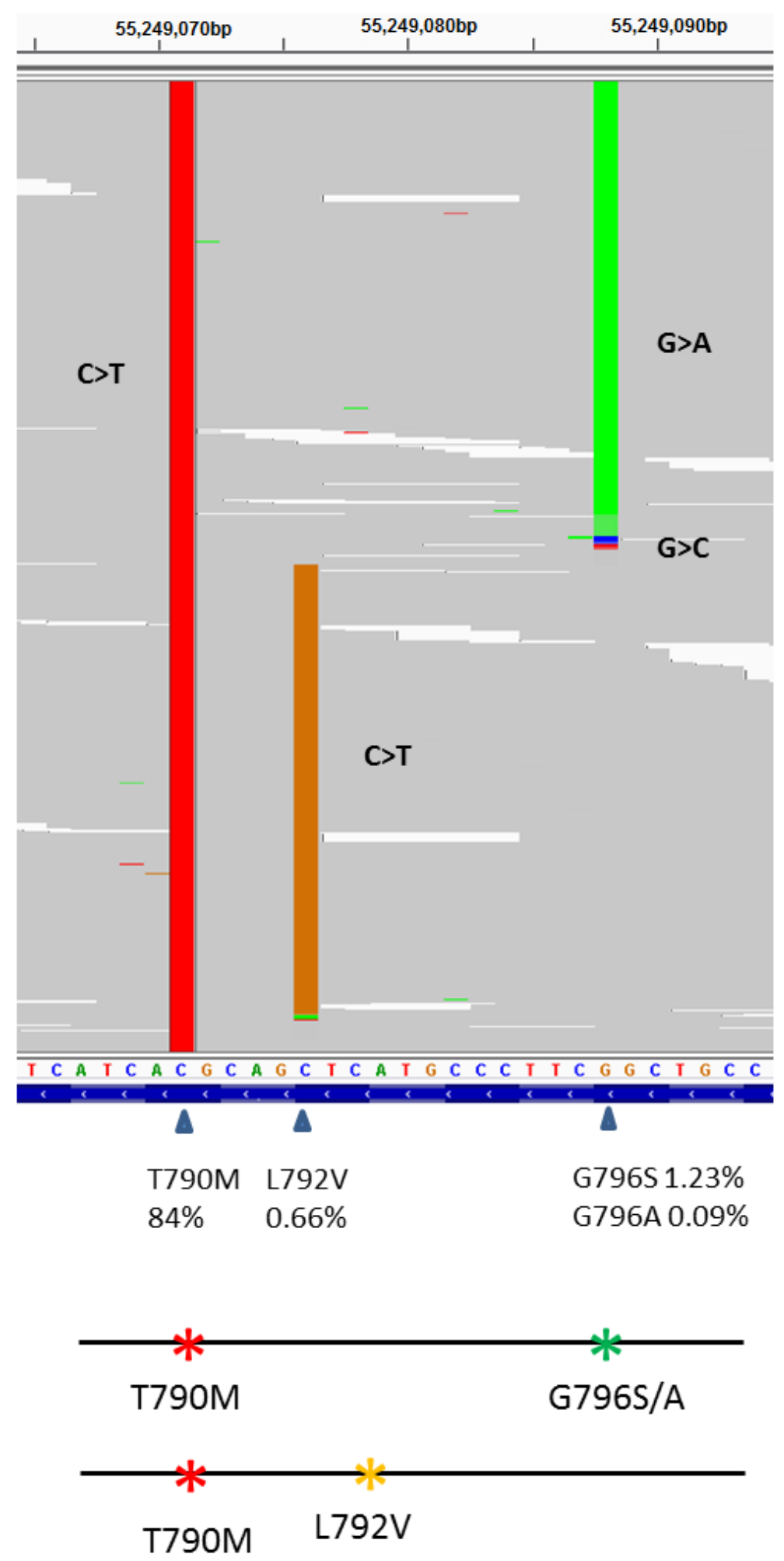

Figure 2: Sequence traces for EGFR L858R and T790M mutations obtained from tumor samples of the patient. The integrated genome viewer revealed the acquired drug resistance mutations against T790M; L792 or G796 in cis with T790M; L792 and G796 are in trans with each other.

known mutations to resist $3^{\text {rd }}$ TKI. At the same time some unknown mutations such as G729V, P936S/A/T, D1014V, R334H, S1081N, R677H were discovered. L792V and G796A/S are in trans. However all mutations are in cis with T790M (Figure 2). Molecular modeling predicted G729V and D1014V could be acquired osimertinib resistance mutations (Figure 3). 


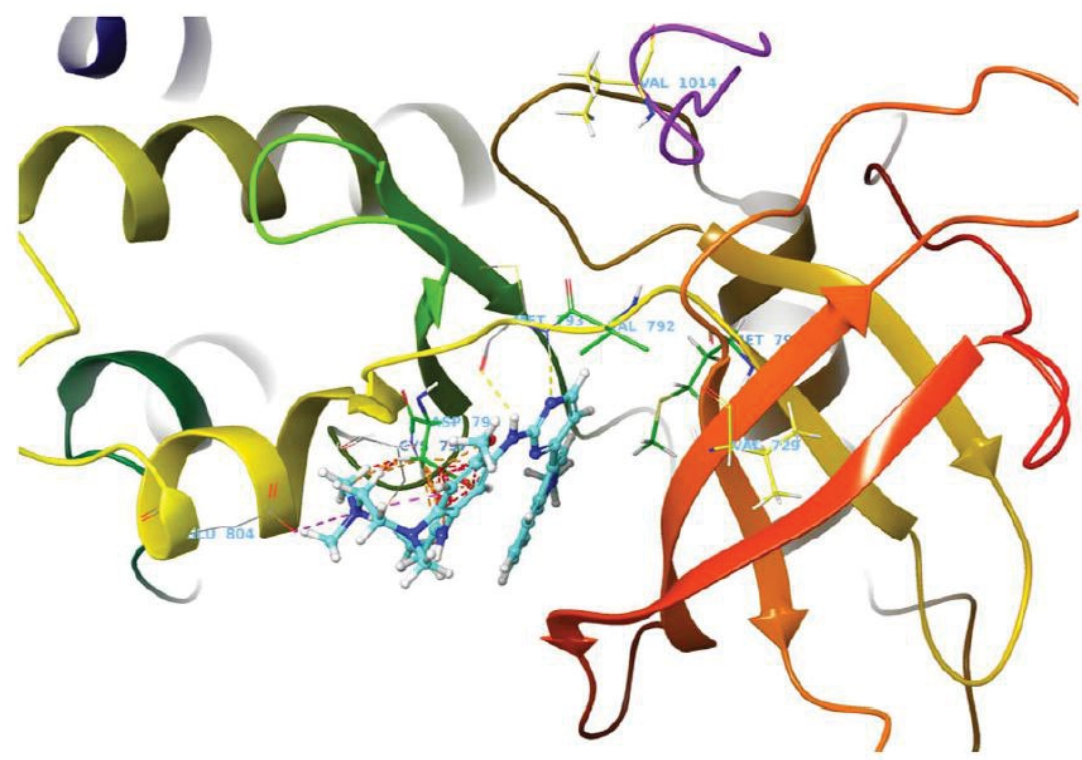

Figure 3: 3D modeling highlights the relevant mutations for AZD9291 resistance. 3D-structure of EGFR mutations interact with osimertinib. The light blue residues were the original mutations, L858R and T790M. The green ones are within the ATP binding pocket which could be the major drivers for the resistance and two yellow mutations: G729V and D1014V could be the secondary drivers for the resistance.

\section{Discussion}

De novo T790M mutation was considered as a well-known primary resistant mechanism to EGFR first generation TKI $[3,5,11]$ and is about $1 \%$ to $8 \%$ of NSCLC patients with an activating EGFR mutation [12]. One meta-analysis result shows that primary (pretreatment) T790M mutation was associated with worse PFS and OS in patients with advanced NSCLC treated with $1^{\text {st }}$ EGFR-TKIs [13]. However, Roseall et al. reported that Low BRCA1 levels neutralized the negative effect of the T790M mutation and were associated with longer progressionfree survival to erlotinib [9]. In the Iressa Pan-Asia Study (IPASS), 11 patients were identified to have primary T790M mutations using an ARMS PCR. Of 11 patients, 7 had concurrent activating EGFR mutations, and 4 of these received gefitinib. Three demonstrated a partial response, while one had stable disease, suggesting that patients with double mutations were sensitive to first generation TKI [14]. In this case, the patient with T790M and L858R mutations was initially treated with erlotinib and had 7-month PFS. T790M mutation clone was very low compared to sensitive L858R mutation in the primary tissue, while $\mathrm{T} 790 \mathrm{M}$ clones were expanded after progression showed by Arms PCR.

Then the patient become quickly resistant to the third-generation TKI osimertinib. We extrapolated that low osimertinib dosage might be one of the possible reasons for osimertinib primary resistance, especially in this case EGFR amplification was detected in patient ctDNA samples. In the AURA study, sixty treatment-naive patients with locally advanced or metastatic EGFRm NSCLC received osimertinib 80 or $160 \mathrm{mg}$ once daily (30 patients per cohort). We can see that Overall ORR was $67 \%$ (95\%CI, $47 \%$ to $83 \%)$ in the $80 \mathrm{mg}$ group, $87 \%$ ( $95 \%$ CI, $69 \%$ to $96 \%$ ) in the $160 \mathrm{mg}$ group, suggest that low osimertinib dosage has lower ORR [15]. The well known variants to resist to EGFR $3^{\text {rd }}$ TKI are C797S, L798I, L692V, E709K mutation and ERBB2, MET amplification [16-18]. Several case reports also showed some new EGFR resistant mutations like G796D [19], G724S [20], L718Q [21]. Besides these major resistant mutations, multi-clone heterogeneous resistance was also reported [22,23]. In this patient, ctDNA assay detected several known osimertinib resistant mutations such as EGFR G796A/S, L792V. Molecular modeling demonstrated that G796A/S directly clashes (red lines) into the methoxybenzene ring of osimertinib while L792V clashes into the V742 of the VAIK motif and destabilizes the ATP binding pocket (Figure 3). Thus the green ones (T790M, G796A/S, L792V) within the pocket could be the major drivers for the drug resistance $[19,22]$; while two yellow ones (G729V and $\mathrm{D} 1014 \mathrm{~V}$ ) are line on the boundary, could be the secondary drivers for the drug resistance. As for L718V/R, these mutations have been reported as a resistance mutations identified in patients who progressed on third-generation EGFR TKI [21]. Transformation to SCLC has also been described recently as a resitant mechanism during osimertinib treatment in at lease 7 clincial cases $[4,24]$. TP53 and RB1 double loss of function mutation is a common molecular pattern in SCLC, while in our case, we did not see clear evidence of TP53 and RB1 double loss of function mutation or SCLC pathology evidence.

After developing osimertinib resistancy, this patient still benefited from Pemetrexed/Cisplatin (PP) chemotherapy for 3 months. Multiple minor MAF unknown significance mutations were listed in this patient while all the clinical treatments were mostly depleted. The heterogeneity of EGFR mutations at the later stage may attribute to her resistance to all available treatments. Findings in this report are promising for applying multidisciplinary approach to treating cancer patient in clinical practice.

\section{Conclusion}

This report provides insight into a successful empirical treatment with erlotinib for a lung adenocarcinoma patient in the presence of pretreatment T790M at small colony. Furthermore, this case report a primary resistant of osimertinib treatment with T790M and L858R double mutation. After osimertinib resistance, the patient received clinical benefit for three months with Pemetrexed/Cisplatin chemotherapy in the situation that no further treatment is available. 
Citation: Zhao X, Dong H, Wang Z, Zhang L, Wang L, et al. (2018) EGFR Primary T790M and L858R Double Mutation Confers Clinical Benefit to Erlotinib and Resistance to Osimertinib in One Lung Adenocarcinoma Patient: A Case Report. J Cancer Sci Ther 10: 366-370. doi: 10.4172/1948-5956.1000569

\section{Ethics Approval and Consent to Participate}

The study was reviewed and approved by the "The First Affiliated hospital of Xi' an Jiaotong University Ethics committee", and the project implementation process was in line with the ethical principples. The patient provided written informed consent to participate in the study.

\section{Consent for publication}

We obtained written informed consent of the patient for the publication of the case report and accompanying images.

\section{Competing interests}

Yizhou Ye, Hua Dong, Lin Shi, Dalei Wang and Fugen Li are employees of 3DMed, Inc. Zheng Wang is employee of AstraZeneca China. The remaining authors declare no competing interest.

\section{Availability of data and material}

The data generated and/or analyzed during this study are included in this published article and the additional files.

\section{Funding}

Not applicable

\section{Authors' Contributions}

$\mathrm{ZX}, \mathrm{DH}$ and YY are responsible for generating and analyzing the data, and drafting the manuscript; $\mathrm{ZL}, \mathrm{WL}$ and $\mathrm{CZ}$ for patient sample collection; WZ, SL and WD for related data confirmation and interpretation; YJ, LP and ZX for pathology review of the patient tissues; YY, DH, LF and YJ for study design and technical discussion; drafting and finalizing the manuscript. All authors have read and approved the final version.

\section{References}

1. Goss G, Tsai CM, Shepherd FA, Bazhenova L, Lee JS, et al. (2016) Osimertinib for pretreated EGFR Thr790Met-positive advanced non-small-cell lung cancer (AURA2): A multicentre, open-label, single-arm, phase 2 study. Lancet Oncol 17: $1643-1652$

2. Mok TS, Wu YL, Ahn MJ, Garassino MC, Kim HR, et al. (2017) Osimertinib or platinum-pemetrexed in EGFR T790M-positive lung cancer. N Engl J Med 376: 629-640.

3. Costa DB, Kobayashi SS (2015) Whacking a mole-cule: Clinical activity and mechanisms of resistance to third generation EGFR inhibitors in EGFR mutated lung cancers with EGFR-T790M. Transl Lung Cancer Res 4: 809-815.

4. Minari R, Bordi P, Del Re M, Facchinetti F, Mazzoni F, et al. (2018) Primary resistance to osimertinib due to SCLC transformation: Issue of T790M determination on liquid re-biopsy. Lung Cancer 115: 21-27.

5. Stewart EL, Tan SZ, Liu G, Tsao MS (2015) Known and putative mechanisms of resistance to EGFR targeted therapies in NSCLC patients with EGFR mutations: A review. Transl Lung Cancer Res 4: 67-81.

6. Suda K, Rivard CJ, Mitsudomi T, Hirsch FR (2017) Overcoming resistance to EGFR tyrosine kinase inhibitors in lung cancer, focusing on non-T790M mechanisms. Expert Rev Anticancer Ther 17: 779-786.

7. Tanimoto A, Takeuchi S, Arai S, Fukuda K, Yamada T, et al. (2017) Histone deacetylase 3 inhibition overcomes BIM deletion polymorphism-Mediated osimertinib resistance in EGFR-Mutant lung cancer. Clin Cancer Res 23: 31393149.
8. Costa DB (2016) Kinase inhibitor-responsive genotypes in EGFR mutated lung adenocarcinomas: Moving past common point mutations or indels into uncommon kinase domain duplications and rearrangements. Transl Lung Cancer Res 5: 331-337.

9. Rosell R, Molina MA, Costa C, Simonetti S, Gimenez-Capitan A, et al, (2011) Pretreatment EGFR T790M mutation and BRCA1 mRNA expression in erlotinib-treated advanced non-small-cell lung cancer patients with EGFR mutations. Clin Cancer Res 17: 1160-1168.

10. Fujita $Y$, Suda K, Kimura H, Matsumoto K, Arao T, et al. (2012) Highly sensitive detection of EGFR T790M mutation using colony hybridization predicts favorable prognosis of patients with lung cancer harboring activating EGFR mutation. J Thorac Oncol 7: 1640-1644.

11. Zhong J, Li L, Wang Z, Bai H, Gai F, et al. (2017) Potential resistance mechanisms revealed by targeted sequencing from lung adenocarcinoma patients with primary resistance to epidermal growth factor receptor (EGFR) tyrosine kinase inhibitors (tkis). J Thorac Oncol 12: 1766-1778.

12. Yu HA, Arcila ME, Hellmann MD, Kris MG, Ladanyi M, et al. (2014) Poor response to erlotinib in patients with tumors containing baseline EGFR T790M mutations found by routine clinical molecular testing. Ann Oncol 25: 423-428.

13. Liu Y, Sun L, Xiong ZC, Sun X, Zhang SL, et al. (2017) Meta-analysis of the impact of de novo and acquired EGFR T790M mutations on the prognosis of patients with non-small cell lung cancer receiving EGFR-tkis. Onco Targets Ther 10: 2267-2279.

14. Fukuoka $\mathrm{M}$, Wu $\mathrm{YL}$, Thongprasert $\mathrm{S}$, Sunpaweravong $\mathrm{P}$, Leong SS, et al (2011) Biomarker analyses and final overall survival results from a phase III, randomized, open-label, first-line study of gefitinib vs. carboplatin/paclitaxel in clinically selected patients with advanced non-small-cell lung cancer in Asia (IPASS). J Clin Oncol 29: 2866-2874.

15. Ramalingam SS, Yang JC, Lee CK, Kurata T, Kim DW, et al. (2018) Osimertinib as first-Line treatment of EGFR mutation-Positive advanced non-small-cell lung cancer. J Clin Oncol 6: 841-849.

16. Kenneth ST, Cloud PP, Enriqueta F, Byoung CC, Daniel S, et al. (2015) Acquired EGFR C797S mutation mediates resistance to AZD9291 in non-small cell lung cancer harboring EGFR T790M. Nat Med 21: 560-562.

17. Chabon JJ, Simmons AD, Lovejoy AF, Esfahani MS, Newman AM, et al. (2016) Circulating tumour DNA profiling reveals heterogeneity of EGFR inhibitor resistance mechanisms in lung cancer patients. Nat Commun 7: 11815

18. Santarpia M, Liguori A, Karachaliou N, Gonzalez-Cao M, Daffinà MG, et al. (2017) Osimertinib in the treatment of non-small-cell lung cancer: Design, development and place in therapy. Lung Cancer (Auckl) 8: 109-125.

19. Zheng D, Hu M, Bai Y, Zhu X, Lu X, et al. (2017) EGFR G796D mutation mediates resistance to osimertinib. Oncotarget 8: 49671-49679.

20. Oztan A, Fischer S, Schrock AB, Erlich RL, Lovly CM, et al. (2017) Emergence of EGFR G724S mutation in EGFR-mutant lung adenocarcinoma post progression on osimertinib. Lung Cancer 111: 84-87.

21. Bersanelli M, Minari R, Bordi P, Gnetti L, Bozzetti C, et al. (2016) L718Q mutation as new mechanism of acquired resistance to AZD9291 in EGFRmutated NSCLC. J Thorac Oncol 11: e121-123.

22. Ou SI, Cui J, Schrock AB, Goldberg ME, Zhu VW, et al. (2017) Emergence of novel and dominant acquired EGFR solvent-front mutations at Gly796 (G796S/R) together with C797S/R and L792F/H mutations in one EGFR (L858R/T790M) NSCLC patient who progressed on osimertinib. Lung Cancer 108: 228-231.

23. Chen K, Zhou F, Shen W, Jiang T, Wu X, et al. (2017) Novel mutations on EGFR Leu792 potentially correlate to acquired resistance to osimertinib in advanced NSCLC. J Thorac Oncol 12: e65-e68.

24. Ham JS, Kim S, Kim HK, Byeon S, Sun JM, et al. (2016) Two cases of smal cell lung cancer transformation from EGFR mutant adenocarcinoma during AZD9291 treatment. J Thorac Oncol 11: e1-e4. 